\title{
Next-to-leading order light-front three-body dynamics
}

\section{J. A. O. Marinho,}

Instituto Tecnológico de Aeronáutica, 12.228-900 São José dos Campos, SP, Brazil

E-mail: adnei@ita.br

\section{T. Frederico}

Instituto Tecnológico de Aeronáutica, 12.228-900 São José dos Campos, SP, Brazil

E-mail: tobias@ita.br

\begin{abstract}
The quasi-potential approach is used to project a four-dimensional field-theoretical model of a three-particle system to the light-front (LF) valence sector. The starting point is the Bethe-Salpeter equation (BSE) for three particles in the ladder approximation. The projection technique allows to eliminate three-body reducible diagrams from the kernel of the LF integral equation for the valence wave function. We construct the next-to-leading order contribution to the kernel of the LF three-body integral equation. We exemplify the method for a three-boson system with a contact interaction.
\end{abstract}

LIGHT CONE 2008 Relativistic Nuclear and Particle Physics

July 7-11 2008

Mulhouse, France

${ }^{*}$ The author thanks the Brazilian agency FAPESP for the financial support. Contract number 03/08470-4.

'Speaker. 


\section{Introduction}

A relativistic framework to treat three-body systems is necessary for our understanding of the valence structure of baryons in terms of constituent quarks. One possible relativistic approach is the quantization on the light-front (LF) hypersurface [1]. To investigate the baryon structure with electroweak probes and large momentum transfers, the covariance of LF wave functions under kinematical boosts is essential [1]. In this context, it is worthwhile to control the covariant properties of the adopted LF framework and its relation to a four-dimensional description [2].

In order to investigate the structure of the baryon LF valence wave function one has to deal with a relativistic three-body problem. Starting from a four-dimensional three-body Bethe-Salpeter equation (BSE), the Faddeev decomposition has to be introduced and the LF projection defined with the correspondent expansion in the Fock space. So far, even the treatment of light-front dynamics (LFD) at leading order (LO) constitutes an involved problem, because a systematic expansion to construct LF three-body equations from a given covariant dynamics is still lacking.

The quasi-potential approach (QPA) offers one possible solution to the problem, by supplying a systematic framework to construct the light-front dynamics of composite systems from a given 4-dimensional model. The approach avoids subtle problems, such as the double-counting of LF reducible graphs (see e.g. [2]), and the spurious divergences in the transverse momenta from the LF projection of the two-fermion box diagram [3,4]. It also treats consistently the fermionic LF instantaneous terms. The QPA is also useful in the study of the electromagnetic structure of composite systems with conserved current operators, within LFD, for two bosons [5] or two fermions [6]. The next step is to apply the QPA to define the light-front dynamics of a 3-body system.

In this contribution we apply the QPA to the four-dimensional three-body BS equation in order to provide the systematical expansion of the kernel of integral equation for LF valence wave function. We show how the QPA works in the study of relativistic three-body systems by going beyond the LO kernel of the LF integral equation. We derive the corresponding next-to-leading order (NLO) contribution to the kernel. We exemplify the method in a three-boson system with a contact interaction by starting from the ladder approximation of the covariant BS equation. The LF bound state equation for the valence wave function in LO and NLO will be obtained. Previous works $[7,8,9,10]$ were limited only to the LO kernel.

\section{QP Expansion and Faddeev Decomposition}

We will restrict ourselves to the ladder BSE for three bosons. Formally the potential in the four-dimensional equation is built by multiplying the two-body interaction $V_{j k}^{(2)}$ from the exchange of a quantum between the particles $i$ and $j$ and by the inverse of the individual propagator of the spectator particle $i, S_{i}$ :

$$
V=\sum_{i=1}^{3} V_{i} ; \quad V_{i}=V_{(2) j k} S_{i}^{-1}
$$

This approximation does not take into account three-body irreducible crossed ladders, self energies, vertex corrections and irreducible 4-dimensional three-body interactions. In terms of the quasipotential approach, the BSE for the transition matrix $T=V+V G_{0} T$, is substituted by a modified 
one, $T=W+W \widetilde{G}_{0} T$. This implies the choice of an auxiliary Green's function $\left(\widetilde{G}_{0}\right)$. Here $G_{0}$ is the free four-dimensional three-body Green's function. To keep the dynamical content of the original BSE, the quasi-potential $W$ should be expressed in terms of the interaction $V$ as $W=V+V \Delta_{0} W$, where $\Delta_{0}:=G_{0}-\widetilde{G}_{0}$.

The QPA is a useful tool to project a given four-dimensional dynamics onto the light-front hypersurface when a particular choice of the auxiliary Green's function is made [2], $\tilde{G}_{0}:=G_{0}\left|g_{0}^{-1}\right| G_{0}$, where $g_{0}=\left|G_{0}\right|$ is the free light-front resolvent, including phase-space factors. The "bar" operation on the right or on the left of a four-dimensional matrix element means that the integration over $k^{-}=k^{0}+k^{3}$ is performed. These integrations eliminate the relative light-front time between the particles. For three-body systems, taking into account four-momentum conservation, only two single particle momenta are independent, therefore the elimination of the relative LF time requires the definition of a LF time projection operation according to

$$
\mid A:=\int d k_{1}^{-} d k_{2}^{-}\left\langle k_{1}^{-} k_{2}^{-}|A, \quad A|:=\int d k_{1}^{-} d k_{2}^{-} A \mid k_{1}^{-} k_{2}^{-}\right\rangle,
$$

with $A$ being an operator that has matrix elements depending on two independent momenta after the center of mass motion is factorized.

In order to make the practical procedure clear, let us write explicitly the three-free Green's function:

$$
\begin{aligned}
\left\langle k_{1}^{-}, k_{2}^{-}\left|G_{0}\right| k_{1}^{\prime-}, k_{2}^{\prime-}\right\rangle= & \frac{-i}{(2 \pi)^{2}} \frac{\delta\left(k_{1}^{-}-k_{1}^{\prime-}\right)}{\hat{k}_{1}^{+} \hat{k}_{2}^{+}\left(K^{+}-\hat{k}_{1}^{+}-\hat{k}_{2}^{+}\right)\left(k_{1}^{-}-\hat{k}_{1 o n}^{-}\right)} \times \\
& \frac{\delta\left(k_{2}^{-}-k_{2}^{\prime-}\right)}{\left(k_{2}^{-}-\hat{k}_{2 o n}^{-}\right)\left(K^{-}-k_{1}^{-}-k_{2}^{-}-\left(K-\hat{k}_{1}-\hat{k}_{2}\right)_{o n}^{-}\right)} .
\end{aligned}
$$

The LF projection yields the LF free Green's function as

$$
g_{0}\left(\underline{k}_{1}, \underline{k}_{2}\right)=\frac{i \theta\left(K^{+}-k_{1}^{+}-k_{2}^{+}\right) \theta\left(k_{1}^{+}\right) \theta\left(k_{2}^{+}\right)}{k_{1}^{+} k_{2}^{+}\left(K^{+}-k_{1}^{+}-k_{2}^{+}\right)\left(K^{-}-k_{1 o n}^{-}-k_{2 o n}^{-}-\left(K-k_{1}-k_{2}\right)_{o n}^{-}\right)},
$$

where, for sake of simplicity, we set $\underline{k} \equiv\left(k^{+}, \vec{k}_{\perp}\right)$. The reader is addressed to refs.[2, 3, 5, 6] to follow the details of the formal manipulations within QPA to perform the light-front projection.

In the 3-body context, the reduced or three-dimensional integral equation for the LF transition matrix is $t=w+w g_{0} t$, which has a Lippman-Schwinger form analogous to the two-body one. The effective interaction $w$ comes from the projection of the quasi-potential $W$ to equal light-front times. We now perform the Faddeev decomposition [11] of the transition matrix. Hence, we write $t_{i}=w_{i}+w_{i} g_{0} t$, with $t=\sum_{i=1}^{3} t_{i}$ and $w_{i}=g_{0}^{-1}\left|G_{0} W_{i} G_{0}\right| g_{0}^{-1}$. This decomposition follows from a corresponding decomposition of the 4-dimensional $W=\sum_{i=1}^{3} W_{i}$.

The Faddeev decomposition of $W=V+V \Delta_{0} W$ is due to $V=\sum_{i=1}^{3} V_{i}$ resulting in $W_{i}=V_{i}+$ $V_{i} \Delta_{0} W$. The integral equation for the Faddeev component of the quasi-potential is derived from $W_{i}=V_{i}+V_{i} \Delta_{0}\left(W_{i}+W_{j}+W_{k}\right)$. It can be rewritten as $\left(1-V_{i} \Delta_{0}\right) W_{i}=V_{i}+V_{i} \Delta_{0}\left(W_{j}+W_{k}\right)$. Inverting the operator on the left side of $W_{i}$, one has that $W_{i}=W_{(2) i}+W_{(2) i} \Delta_{0}\left(W_{j}+W_{k}\right)$, where $W_{(2) i}=$ $V_{i}+V_{i} \Delta_{0} W_{(2) i}$ is the quasi-potential for the two-body system with particle $i$ as a spectator.

The expansion in powers of $V$ of $W_{i}$ is given by:

$$
W_{i}=V_{i}+V_{i} \Delta_{0}\left(V_{i}+V_{j}+V_{k}\right)+V_{i} \Delta_{0}\left(V_{i}+V_{j}+V_{k}\right) \Delta_{0}\left(V_{i}+V_{j}+V_{k}\right)+\ldots
$$


The LO and NLO terms are given by $W_{i}^{L O}=V_{i}$ and by $W_{i}^{N L O}=V_{i}+V_{i} \Delta_{0}\left(V_{i}+V_{j}+V_{k}\right)$, respectively. Making the expansion of the Faddeev equations for $W_{i}$, only connected terms in the two-body subsystem quasi-potential appear:

$$
W_{i}=W_{(2) i}+W_{(2) i} \Delta_{0}\left(W_{(2) j}+W_{(2) k}\right)+W_{(2) i} \Delta_{0} W_{(2) j} \Delta_{0}\left(W_{(2) i}+W_{(2) k}\right)+\ldots .
$$

The Faddeev components of LF effective potential in LO and NLO are written in terms of the above expansion as

$$
w_{i}^{L O}=g_{0}^{-1}\left|G_{0} V_{i} G_{0}\right| g_{0}^{-1}, \quad w_{i}^{N L O}=w_{i}^{L O}+g_{0}^{-1}\left|G_{0} V_{i} \Delta_{0}\left(V_{i}+V_{j}+V_{k}\right) G_{0}\right| g_{0}^{-1},
$$

respectively. In the next section we will show in an example the explicit expressions for the threeboson LF bound state equations in LO and NLO.

\section{Three-boson model with zero range interaction}

The interaction Lagrangian for a zero range model can be written as $\mathscr{L}_{I}=\frac{1}{4 !} \lambda \phi^{4}$. Following the prescription given by Eq.(2.1), we have for the potential $V_{i}$ :

$$
\left\langle k_{j}, k_{k}\left|V_{i}\right| k_{j}^{\prime}, k_{k}^{\prime}\right\rangle=\lambda(2 \pi)^{2} \delta^{4}\left(k_{i}-k_{i}^{\prime}\right)\left(k_{i}^{2}-m^{2}\right) .
$$

For the contact interaction, we can easily demonstrate the property $V_{i} \Delta_{0} V_{i}=0$. Therefore, we have that $W_{(2) i}=V_{i}$, which allows to write the integral equation for the Faddeev component of the quasipotential as $W_{i}=V_{i}+V_{i} \Delta_{0}\left(W_{j}+W_{k}\right)$. The expansion of the this equation gives the LO and the NLO quasi-potential as: $W_{i}^{L O}=V_{i}$ and $W_{i}^{N L O}=W_{i}^{L O}+V_{i} \Delta_{0}\left(V_{j}+V_{k}\right)$, respectively.

\subsection{LO three-boson LF dynamics}

The effective potential in LO is $w_{i}^{L O}=g_{0}^{-1}\left|G_{0} V_{i} G_{0}\right| g_{0}^{-1}$, which has matrix elements given by:

$$
w_{i}^{L O}\left(\underline{k}_{j}, \underline{k}_{k} ; \underline{k}_{j}^{\prime}, \underline{k}_{k}^{\prime}\right)=-2 \pi i \lambda k_{i}^{+} \delta^{3}\left(\underline{k}_{i}-\underline{k}_{i}^{\prime}\right) .
$$

The equation for the Faddeev components of the bound-state vertex in is given by $v_{i}=w_{i} g_{0}\left(v_{i}+\right.$ $\left.v_{j}+v_{k}\right)$ which in LO gives that $v_{i}^{L O}=\left(1-w_{i}^{L O} g_{0}\right)^{-1} w_{i}^{L O} g_{0}\left(v_{j}^{L O}+v_{k}^{L O}\right)$. Introducing the matrix elements of $w_{i}^{L O}$ and $g_{0}$ in the above equation one gets:

$$
v_{i}^{L O}\left(\underline{k}_{j}, \underline{k}_{k}\right)=2 \int d^{3} \underline{k}_{i}^{\prime} d^{3} \underline{k}_{j}^{\prime}\left\langle\underline{k}_{j}, \underline{k}_{k}\left|\left(1-w_{i}^{L O} g_{0}\right)^{-1} w_{i}^{L O}\right| \underline{k}_{i}^{\prime}, \underline{k}_{j}^{\prime}\right\rangle g_{0}\left(\underline{k}_{i}^{\prime}, \underline{k}_{j}^{\prime}\right) v_{k}^{L O}\left(\underline{k}_{i}^{\prime}, \underline{k}_{j}^{\prime}\right),
$$

where the factor 2 comes from the symmetry of the total vertex function by the exchange of the bosons. In detail

$$
\left\langle\underline{k}_{j}, \underline{k}_{k}\left|\left(1-w_{i}^{L O} g_{0}\right)^{-1} w_{i}^{L O}\right| \underline{k}_{i}^{\prime}, \underline{k}_{j}^{\prime}\right\rangle=i \tau\left(M_{j k}^{2}\right) k_{i}^{+} \delta^{3}\left(\underline{k}_{i}-\underline{k}_{i}^{\prime}\right),
$$

where $M_{j k}^{2}=\left(K-k_{i o n}\right)^{2}$. Explicitly one has that the two-boson amplitude is given by

$$
\tau\left(M_{j k}^{2}\right)=2 \pi\left[\lambda^{-1}+\frac{1}{8 \pi^{2}} \int_{0}^{1} d x \int d^{2} k_{\perp} \frac{1}{x(1-x)\left(M_{j k}^{2}-\frac{k_{\perp}^{2}+m^{2}}{x(1-x)}\right)}\right]^{-1} .
$$


The integral equation for the Faddeev component of the three-boson vertex becomes:

$$
v_{i}^{L O}\left(\underline{k}_{j}, \underline{k}_{k}\right)=-2 \tau\left(M_{j k}^{2}\right) \int d^{3} \underline{k}_{j}^{\prime} \frac{\theta\left(k_{j}^{\prime+}\right) \theta\left(K^{+}-k_{i}^{+}-k_{j}^{\prime+}\right) v_{k}^{L O}\left(\underline{k}_{i}, \underline{k}_{j}^{\prime}\right)}{k_{j}^{\prime+}\left(K^{+}-k_{i}^{+}-k_{j}^{\prime+}\right)\left(K^{-}-k_{\text {ion }}^{-}-k_{j o n}^{\prime-}-\left(K-k_{i}-k_{j}^{\prime}\right)_{\text {on }}^{-}\right)},
$$

where the elementary volume is $d^{3} \underline{k}=d k^{+} d^{2} k_{\perp} /\left(2(2 \pi)^{3}\right)$. Note that the dependence of left hand side of Eq. (3.6) depends only on $\underline{k}_{i}$, therefore we can write that $v_{i}^{L O}\left(\underline{k}_{j}, \underline{k}_{k}\right) \equiv v_{i}^{L O}\left(\underline{k}_{i}\right)$, and making the transformation of the integration momentum from $\underline{k}_{j}^{\prime}$ to $\underline{k}_{k}^{\prime}$, owing to $\underline{k}_{j}^{\prime}=\underline{K}-\underline{k}_{k}^{\prime}-\underline{k}_{i}$, one obtain the LO three-body bound state equation derived in ref.[7]. In that work, a regularization in the transverse momentum of the spectator particle was used. In ref.[9], the cutoff was not introduced and it was discovered by numerical calculations that the three-boson bound states exist in a range of values of two-boson bound state masses.

\subsection{NLO three-boson LF dynamics}

We deduce now the contribution to the kernel of the integral equation for the bound-state at next-to-leading order. The QP in NLO is $W_{i}^{N L O}=W_{i}^{L O}+V_{i} \Delta_{0}\left(V_{j}+V_{k}\right)$ and the corresponding effective potential is:

$$
w_{i}^{N L O}=w_{i}^{L O}+\Delta w_{i}^{N L O}, \quad \Delta w_{i}^{N L O}=g_{0}^{-1}\left|G_{0} V_{i} \Delta_{0} V_{j} G_{0}\right| g_{0}^{-1}+g_{0}^{-1}\left|G_{0} V_{i} \Delta_{0} V_{k} G_{0}\right| g_{0}^{-1} .
$$

The integral equation for the Faddeev component can be written as:

$$
v_{i}^{N L O}=\left(1-w_{i}^{L O} g_{0}\right)^{-1} w_{i}^{L O} g_{0} \sum_{n \neq i} v_{n}^{N L O}+\left(1-w_{i}^{L O} g_{0}\right)^{-1} \Delta w_{i}^{N L O} g_{0} \sum_{n} v_{n}^{N L O},
$$

where the first term in the right hand side is analogous to Eq. (3.6). The second term is connected and it gives the NLO contribution to the three-boson light-front bound-state equation:

$$
\begin{aligned}
& v_{i}^{N L O}\left(\underline{k}_{j}, \underline{k}_{k}\right)=-\tau\left(M_{j k}^{2}\right)\left(2 \int d^{3} \underline{k}_{j}^{\prime} \frac{\theta\left(k_{i}^{+}\right) \theta\left(k_{j}^{\prime+}\right) \theta\left(K^{+}-k_{i}^{+}-k_{j}^{\prime+}\right) v_{k}^{N L O}\left(\underline{k}_{i}, \underline{k}_{j}^{\prime}\right)}{k_{j}^{+} k_{k}^{\prime+}\left(K^{-}-k_{\text {ion }}^{-}-k_{j o n}^{\prime-}-\left(K-k_{i}-k_{j}^{\prime}\right)_{\text {on }}^{-}\right)}+\right. \\
& 2 \pi \lambda \int d^{3} \underline{k}_{j}^{\prime} d^{3} \underline{k}_{k}^{\prime} \frac{\theta\left(k_{i}^{+}-K^{+}+k_{k}^{\prime+}\right) \theta\left(K^{+}-k_{j}^{\prime+}-k_{k}^{\prime+}\right) \theta\left(k_{j}^{\prime+}\right) \theta\left(k_{k}^{\prime+}\right)}{k_{j}^{\prime+} k_{k}^{\prime+}\left(k_{i}^{+}-K^{+}+k_{k}^{\prime+}\right)\left(K^{+}-k_{j}^{\prime+}-k_{k}^{\prime+}\right)} \times \\
& \frac{1}{\left(K^{-}-k_{j o n}^{\prime-}-k_{k o n}^{\prime-}-\left(K-k_{j}^{\prime}-k_{k}^{\prime}\right)_{\text {on }}^{-}\right)} \frac{v_{i}^{N L O}\left(\underline{k}_{j}^{\prime}, \underline{k}_{k}^{\prime}\right)+v_{j}^{N L O}\left(\underline{k}_{k}^{\prime}, \underline{k}_{i}^{\prime}\right)+v_{k}^{N L O}\left(\underline{k}_{i}^{\prime}, \underline{k}_{j}^{\prime}\right)}{\left(K^{-}-k_{j o n}^{-}-k_{\text {kon }}^{-}-k_{j o n}^{\prime-}-\left(k_{i}-K+k_{k}^{\prime}\right)_{\text {on }}^{-}-\left(K-k_{j}^{\prime}-k_{k}^{\prime}\right)_{\text {on }}^{-}\right)} \\
& \left.+\left(k_{k}^{\prime} \leftrightarrow k_{j}^{\prime}\right)\right) .
\end{aligned}
$$

The integrand of the second term in the above equation comes from $g_{0}^{-1}\left|G_{0} V_{i} \Delta_{0} V_{k} G_{0}\right| \sum_{n} v_{n}^{N L O}$ while the term indicated by $\left(k_{k}^{\prime} \leftrightarrow k_{j}^{\prime}\right)$ comes from $g_{0}^{-1}\left|G_{0} V_{i} \Delta_{0} V_{k} G_{0}\right| \sum_{n} v_{n}^{N L O}$. In Fig. 1 , the LO and NLO are represented diagrammatically.

The NLO contribution to the kernel of Eq. (3.9) is an irreducible LF three-boson interaction (see also [12]), which comes from the coupling of the valence state to a five-boson intermediate state (see Fig. 1). The virtual five-body system is heavier than the three-boson system leading to an effective interaction that acts at short distances. The solution of of Eq. (3.9) still requires that a renormalization procedure should be applied to the integral equation as the bare coupling constant appears in the NLO kernel. This issue will be not tackled here. 

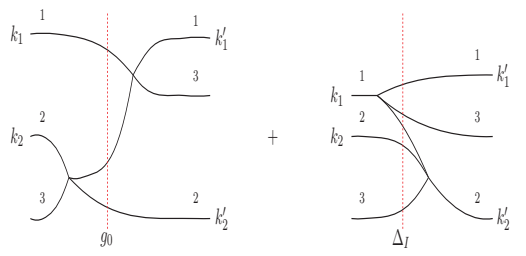

Figure 1: Diagrammatical representation of the LO (left) and NLO (right) contributions to the kernel of the LF integral equation for the Faddeev components of the vertex function of the valence state .

\section{Summary}

We show how to use the quasi-potential approach to project a field-theoretic dynamics of three-particle systems in the ladder approximation to the light-front. Our starting point is the fourdimensional Bethe-Salpeter equation for three particles. The projection technique allows to eliminate three-body reducible diagrams from the kernel of the integral equation for the valence wave function. We applied the Faddeev decomposition to the QP to derive connected equations for the corresponding components. From these components we construct the next-to-leading order contribution to the kernel of the light-front three-body integral equation for the transition matrix. We exemplify the method for a bound three-boson system with a contact interaction deriving explicitly the NLO integral equation for the Faddeev component of the LF vertex function of the valence component of the bound state. Within the same framework we can also derive conserved current operators acting on the valence sector of three-body systems. This problem and the issue of renormalization of the NLO LF three-body equation are left for a future work.

We thank Fundação de Amparo à Pesquisa do Estado de São Paulo and Conselho Nacional de Desenvolvimento Científico e Tecnológico for partial support.

\section{References}

[1] S.J. Brodsky,H.-C. Pauli, S. S. Pinsky, Phys. Rep. 301, 299 (1998).

[2] J. H. O. Sales, T. Frederico, B. V. Carlson, P. U.Sauer, Phys. Rev. C 61, 044003 (2000).

[3] J. H. O. Sales, T. Frederico, B. V. Carlson, P. U.Sauer, Phys. Rev. C 63, 064003 (2001).

[4] B. L. G. Bakker, J. K. Boomsma, C.-R. Ji, Phys. Rev. D 75, 065010 (2007).

[5] J.A.O. Marinho, T. Frederico and P. U. Sauer, Phys. Rev. D 76, 096001 (2007).

[6] J. A. O. Marinho, T. Frederico, G. Salmè, E. Pace and P. U. Sauer, Phys. Rev. D 77, 116010 (2008).

[7] T. Frederico, Phys. Lett. B 282, 409 (1992).

[8] W. R. B. de Araújo, J. P. B. C. de Melo, and T. Frederico, Phys. Rev. C52, 2733 (1995)

[9] J. Carbonell, V.A. Karmanov, Phys.Rev.C 67, 037001 (2003).

[10] M. Beyer, S Mattiello, T. Frederico, H.J. Weber, Few-Body Systems 33, 89 (2003).

[11] L. D. Faddeev, Sov. Phys. JETP 12, 1014 (1960).

[12] V. A. Karmanov and P. Maris, contribution to this Proceedings. 